\title{
Maximum-Likelihood Symbol Synchronization for IEEE 802.11a WLANs in Unknown Frequency-Selective Fading Channels
}

\author{
Yik-Chung Wu, Kun-Wah Yip, Member, IEEE, Tung-Sang Ng, Fellow, IEEE, and \\ Erchin Serpedin, Senior Member, IEEE
}

\begin{abstract}
Based on the maximum-likelihood principle and the preamble structure of IEEE 802.11a wireless local area network (WLAN) standard, this paper proposes a new symbol synchronization algorithm for IEEE 802.11a WLANs over frequencyselective fading channels. In addition to the physical channel, the effects of filtering and unknown sampling phase offset are also considered. Loss in system performance due to synchronization error is used as a performance criterion. Computer simulations show that the proposed algorithm exhibits better performances than the simple correlation-based algorithms. When compared to the algorithm based on the generalized Akaike information criterion, the proposed algorithm presents comparable performance and exhibits reduced complexity.
\end{abstract}

Index Terms-Frequency selective, IEEE 802.11a wireless local area network (WLAN), maximum likelihood (ML), multipath fading channel, symbol synchronization.

\section{INTRODUCTION}

I EEE 802.11a wireless local area networks (WLANs), which support high-speed data transmissions up to $54 \mathrm{Mb} / \mathrm{s}$ [1], employ burst-mode transmission and orthogonal frequency division multiplexing (OFDM) as the transmission technique. Although OFDM is well known for its ability to combat the intersymbol interference (ISI) introduced by multipath channels [2], [3], incorrect positioning of the fast Fourier transform (FFT) window within an OFDM symbol reintroduces ISI during data demodulation, causing serious performance degradation [4], [5]. Symbol synchronization is therefore one of the important tasks performed at receivers in IEEE 802.11a WLANs.

A number of methods for OFDM symbol synchronization have been proposed in the literature. Methods that exploit the periodic structure of cyclic prefixes in OFDM symbols have been proposed in [5]-[7]. Algorithms based on the use of repeated preambles have been reported in [8]-[12] and [14].

Manuscript received July 17, 2003; revised January 20, 2004; accepted September 17, 2004. This work was supported by NSF Award CCR-0092901. The editor coordinating the review of this paper and approving it for publication is A. Swami.

Y.-C. Wu and E. Serpedin are with the Department of Electrical Engineering, Texas A\&M University, College Station, TX 77843-3128 USA (e-mail: ycwu@ee.tamu.edu; serpedin@ee.tamu.edu).

K.-W. Yip is with the Philips Research East Asia-Shanghai, Kerry Everbright City, Shanghai 200070, China (e-mail: stanley.kw.yip@ philips.com).

T.-S. Ng is with the Department of Electrical and Electronic Engineering, The University of Hong Kong, Hong Kong (e-mail: tsng@eee.hku.hk).

Digital Object Identifier 10.1109/TWC.2005.858008
In [4] and [15], additional pilot subcarriers are used to further improve the estimation accuracy after coarse timing synchronization is established by correlation-based methods. Although the techniques of [4]-[15] (which were originally developed for general OFDM systems) may be applied to IEEE 802.11a WLANs, a higher synchronization accuracy can be obtained by using optimized algorithms that take advantage of the known preamble structure located at the beginning of a data packet.

Recently, symbol-synchronization techniques that are specifically designed for IEEE 802.11a WLANs have been reported in [16] and [17]. In [16], the received signal is correlated with a known training-symbol sequence and the absence of the expected correlation peak is detected. Despite the advantage that a simple correlator can be easily implemented at the receiver, its performance is poor in dispersive channels [16], indicating that more sophisticated synchronization algorithms are required. In [17], the generalized Akaike information criterion (GAIC) is used to jointly estimate the channel and establish timing synchronization. Although the reported performance is good, its complexity is extremely high.

In this paper, we develop a maximum-likelihood (ML) symbol synchronizer for IEEE 802.11a WLANs on frequencyselective fading channels. A realistic channel that includes the effects of filtering and sampling time offset in addition to the physical channel with random path delays is considered. Furthermore, the loss in system performance due to synchronization error is used as the performance criterion [5], [27], as opposed to the requirement that the estimated symbol timing has to be within certain limits with respect to a fixed reference point. The proposed algorithm is compared with four existing symbol-synchronization algorithms, one of which is specifically designed for IEEE 802.11a WLANs [17], and three other algorithms for general OFDM frame synchronization [6], [12], [14]. Simulation results indicate that, in general, joint estimation of symbol position and channel (as is the case with the proposed algorithm and the algorithm based on GAIC [17]) gives better performances than the correlation-based algorithms [6], [12], [14]. When compared to the GAIC algorithm [17], the proposed algorithm exhibits comparable performances, but the complexity of the proposed algorithm is much smaller than that of the GAIC algorithm due to the smaller observation length.

We mention that there are also work on general frame synchronization for packet-based transmission over frequencyselective channels (not limited to OFDM systems) [18]-[20]. 

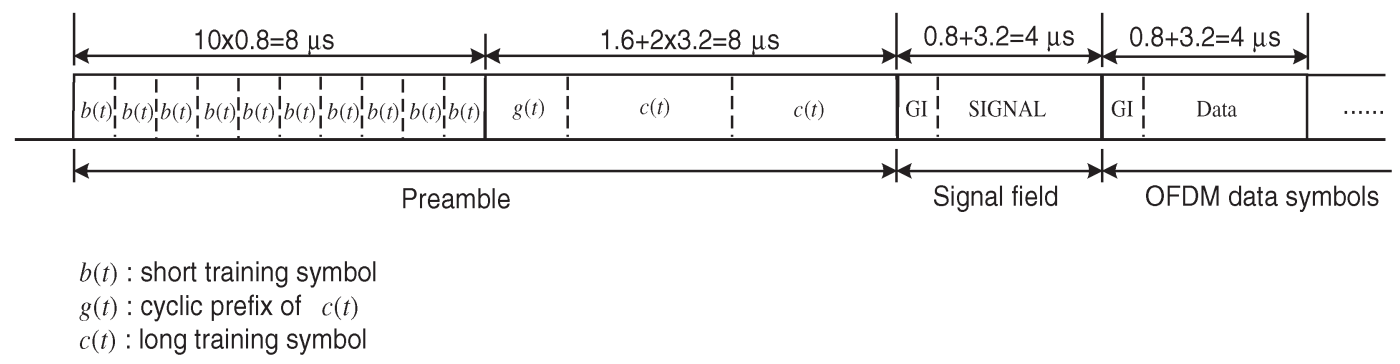

Fig. 1. Packet structure for IEEE 802.11a WLANs.

It will be shown later that part of the proposed synchronization algorithm in this paper reduces to the same detector proposed in [18]-[20] under certain conditions. Note also that the seemingly related synchronization technique for pilot-symbolassisted modulation (PSAM) [26] is not applicable to the problem under consideration since the pilot-symbol insertion mechanism in PSAM is totally different from the preamble structure of a data packet in IEEE 802.11a WLAN.

This paper is organized as follows. Section II describes the received-signal model. The symbol-synchronizationperformance criterion is discussed in Section III. Section IV derives the ML synchronization algorithms for the IEEE 802.11a WLANs. Simulation results on the synchronization performances and comparison with other algorithms are presented in Section V. Finally, conclusions are drawn in Section VI.

\section{RECEIVED-SignAl MODEL}

\section{A. Signal and Channel Models}

Fig. 1 depicts the packet structure used in IEEE 802.11a WLANs. In each packet, the data carrying OFDM symbols are preceded by a preamble, which is used for start-of-packet detection, automatic gain control, symbol timing and frequency synchronization, and channel estimation. The preamble itself consists of two parts. The first part comprises ten short training symbols $b(t)$, each of length $T_{b}=800 \mathrm{~ns}$. In the second part, a cyclic prefix $g(t)$ of length $T_{g}=1.6 \mu \mathrm{s}$ is followed by two long training symbols $c(t)$, each of length $T_{c}=3.2 \mu \mathrm{s}$. The baseband-equivalent model of the preamble is given by [1]

$$
\begin{aligned}
s(t)=\sqrt{2 P}\left\{\sum_{i=0}^{9} b(t-\right. & \left.i T_{b}\right)+g\left(t-10 T_{b}\right) \\
& \left.+\sum_{i=0}^{1} c\left(t-10 T_{b}-T_{g}-i T_{c}\right)\right\}
\end{aligned}
$$

where $P$ is the RF signal power

$$
\begin{aligned}
& b(t) \triangleq \Pi\left(\frac{t}{T_{b}}\right) \times \frac{1}{\sqrt{52}} \sum_{k=-26}^{26} S_{k} \mathrm{e}^{j 2 \pi k \Delta_{\mathrm{f}} t} \\
& g(t) \triangleq \Pi\left(\frac{t}{T_{g}}\right) \times \frac{1}{\sqrt{52}} \sum_{k=-26}^{26} L_{k} \mathrm{e}^{j 2 \pi k \Delta_{\mathrm{f}}\left(t-T_{g}\right)} \\
& c(t) \triangleq \Pi\left(\frac{t}{T_{c}}\right) \times \frac{1}{\sqrt{52}} \sum_{k=-26}^{26} L_{k} \mathrm{e}^{j 2 \pi k \Delta_{\mathrm{f}} t}
\end{aligned}
$$

In (2)-(4), $\Pi(x)$ is a rectangular function giving a value of 1 when $0 \leq x<1$ and 0 otherwise, $\Delta_{\mathrm{f}}=312.5 \mathrm{kHz}$ is the subcarrier separation, $S_{-26: 26}$ and $L_{-26: 26}$ are two trainingsymbol sequences given in [1, eqs. (6) and (8)], respectively. The signal model given by (1) is slightly different from the one specified in the standard [1] in that a raised-cosine window should be used to mask the two parts of the preamble for reducing the spectral sidelobes of transmitted signals. This windowing function is not included in the model considered here in order to simplify derivation of the synchronization algorithm. The raised-cosine window was included in the simulation model during the assessment of synchronization performances.

The signal $s(t)$ is passed through the transmission filter $f_{\mathrm{T}}(t)$, up converted to high frequency, and transmitted through a multipath frequency-selective fading channel. At the receiver, the signal is first passed through the RF filter and then down converted into baseband signal, where additional filtering occurs. Assuming the channel is static over the duration of a packet, the complex envelope of the received and filtered signal is given by

$r(t)=\mathrm{e}^{j 2 \pi v t}\left\{s(t) \star f_{\mathrm{T}}(t) \star \sum_{n=0}^{L_{\mathrm{o}}-1} \gamma_{n} \delta\left(t-\tau_{n}\right) \star f_{\mathrm{R}}(t)\right\}+w(t)$

where $v$ is the carrier frequency offset, $\star$ denotes continuous time convolution, $\gamma_{n}$ is the complex-valued channel coefficients for the $n$th path with $\tau_{n}$ as the path delay, $L_{\mathrm{o}}$ is the total number of physical paths of the multipath channel, $f_{\mathrm{R}}(t)$ is the lowpass equivalent response of the combined RF and baseband filter, and $w(t)$ is the filtered complex Gaussian noise. It is assumed that the channel gain is unity (i.e., $\mathbb{E}\left[\sum_{n=0}^{L_{\circ}-1}\left|\gamma_{n}\right|^{2}\right]=$ 1 , where $\mathbb{E}[\cdot]$ stands for expectation). Furthermore, without loss of generality, it is assumed that $\tau_{0}=0$ since the delay of the first path can be translated to a delay in sampling. Expressing the convolution in the form of integral, the received signal can be rewritten as

$$
r(t)=\mathrm{e}^{j 2 \pi v t} \int_{-\infty}^{\infty} s(t-u)\left[\sum_{n=0}^{L_{\mathrm{o}}-1} \gamma_{n} f\left(u-\tau_{n}\right)\right] d u+w(t)
$$

where $f(t) \triangleq f_{\mathrm{T}}(t) \star f_{\mathrm{R}}(t)$.

Now, the received signal is sampled at $t=k T_{\text {sam }}+\varepsilon_{\mathrm{o}} T_{\text {sam }}$, where $1 / T_{\text {sam }}=20 \mathrm{MHz}$, which is the suggested sampling rate in the standard [1], and $\varepsilon_{\mathrm{o}} \in[0,1)$ is the unknown time offset induced by the combination of the delay of the first path of 
the channel and the sampling phase offset. It follows that the sampled signal is given by

$$
\begin{aligned}
r_{k}= & \mathrm{e}^{j 2 \pi v\left(k T_{\mathrm{sam}}+\varepsilon_{\mathrm{o}} T_{\mathrm{sam}}\right)} \\
& \times \int_{-\infty}^{\infty} s\left(k T_{\mathrm{sam}}+\varepsilon_{\mathrm{o}} T_{\mathrm{sam}}-u\right) \\
& \times\left[\sum_{n=0}^{L_{\mathrm{o}}-1} \gamma_{n} f\left(u-\tau_{n}\right)\right] d u+w_{k} \\
= & \mathrm{e}^{j 2 \pi v\left(k T_{\mathrm{sam}}+\varepsilon_{\mathrm{o}} T_{\mathrm{sam}}\right)} \frac{1}{T_{\mathrm{sam}}} \\
& \times \int_{-\infty}^{\infty} s\left(k T_{\mathrm{sam}}-u^{\prime}\right) h\left(u^{\prime}\right) d u^{\prime}+w_{k}
\end{aligned}
$$

where $r_{k} \triangleq r\left(k T_{\text {sam }}+\varepsilon_{\mathrm{o}} T_{\mathrm{sam}}\right), \quad w_{k} \triangleq w\left(k T_{\mathrm{sam}}+\varepsilon_{\mathrm{o}} T_{\mathrm{sam}}\right)$, and $h(t)$ is the equivalent channel that includes the effects of the transmission filter, physical channel, RF and baseband filtering at the receiver, the timing delay induced by the delay of the first path of the channel, and the sampling phase offset, and is defined as

$$
h(t) \triangleq T_{\text {sam }} \sum_{n=0}^{L_{\mathrm{o}}-1} \gamma_{n} f\left(t-\tau_{n}+\varepsilon_{\mathrm{o}} T_{\mathrm{sam}}\right) .
$$

Notice that the bandwidth (one-sided baseband) of $s(t)$ is $\mathrm{BW}_{s}=(26+1) \Delta_{\mathrm{f}} \approx 8.44 \mathrm{MHz}$ [1] (see also (2)-(4), meaning that $\mathrm{BW}_{s}<1 / 2 T_{\mathrm{sam}}=10 \mathrm{MHz} .{ }^{1}$ According to [24], if the bandwidth of the equivalent channel $h(t)$ [which is equal to the bandwidth of $f(t)$ ] satisfies ${ }^{2}$

$$
\mathrm{BW}_{h}<\frac{1}{T_{\mathrm{sam}}}-\mathrm{BW}_{s}
$$

then by the equivalence of digital and analog filtering for band-limited signals, the sampled received signal can be expressed as

$$
\begin{aligned}
r_{k}=\mathrm{e}^{j 2 \pi v\left(k T_{\mathrm{sam}}+\varepsilon_{\mathrm{o}} T_{\mathrm{sam}}\right)} & \\
& \times \sum_{i=-\infty}^{\infty} s\left(k T_{\mathrm{sam}}-i T_{\mathrm{sam}}\right) h\left(i T_{\mathrm{sam}}\right)+w_{k} .
\end{aligned}
$$

The meaning of (9) is that the filter $f(t)$ can be designed such that its bandwidth is larger than $1 / 2 T_{\text {sam }}$ as long as the aliasing caused by sampling lies outside the passband of signal $s(t)$. A simple example of $f(t)$ that makes (9) satisfied is the raised-

\footnotetext{
${ }^{1}$ Strictly speaking, most of the signals in communications are not band limited, however, notice that the frequency separation between two OFDM data channels is only $20 \mathrm{MHz}$ [1]; taking into account the guard bands, we can treat the one-sided signal bandwidth to be strictly smaller than $10 \mathrm{MHz}$ (or at least the effect of signal outside the "main" bandwidth is so small that we can ignore it).

${ }^{2}$ For practical filters, they can always be designed to have attenuation at the stopband as high as possible (with the cost of complexity), therefore, we can also treat $h(t)$ as band-limited.
}

cosine filter

$$
f_{\mathrm{rc}}(t)=\frac{1}{T_{\mathrm{sam}}} \cdot \frac{\sin \left(\frac{\pi t}{T_{\mathrm{sam}}}\right)}{\frac{\pi t}{T_{\mathrm{sam}}}} \frac{\cos \left(\frac{\pi \beta t}{T_{\mathrm{sam}}}\right)}{1-\frac{4 \beta^{2} t^{2}}{T_{\mathrm{sam}}^{2}}}
$$

with $\beta<0.156$ since it is required that $\mathrm{BW}_{h}=(1+\beta) /$ $2 T_{\text {sam }}<1 / T_{\text {sam }}-\mathrm{BW}_{s}$. The scaling factor $1 / T_{\text {sam }}$ is included in (11) such that the frequency response $F_{\mathrm{rc}}(\omega)=1$ for $|\omega / 2 \pi|<(1-\beta) / 2 T_{\text {sam. }}$. Without loss of generality, we employ both $f_{\mathrm{T}}(t)$ and $f_{\mathrm{R}}(t)$ as square root raised-cosine filters such that $f(t)=f_{\mathrm{T}}(t) \star f_{\mathrm{R}}(t)$ is given by (11) with rolloff factor $\beta=0.1$ for the rest of the paper. Because $f_{\mathrm{R}}(t)$ is a square root cosine filter, the filtered noise samples are uncorrelated with variance $\sigma_{w}^{2}=\mathbb{E}\left[|w(t)|^{2}\right]$.

Remark 1: For the special case if: 1) the path delays are sample spaced (i.e., $\tau_{n}=n T_{\text {sam }}$ ), 2) the timing delay $\varepsilon_{\mathrm{o}}=0$, and 3) $f(t)$ is a raised-cosine pulse with $\beta<0.156$, then

$$
\begin{aligned}
h\left(i T_{\mathrm{sam}}\right) & =T_{\mathrm{sam}} \sum_{n=0}^{L_{\mathrm{o}}-1} \gamma_{n} f_{\mathrm{rc}}\left(i T_{\mathrm{sam}}-n T_{\mathrm{sam}}\right) \\
& =\sum_{n=0}^{L_{\mathrm{o}}-1} \gamma_{n} \delta(i-n)
\end{aligned}
$$

since the values of the raised-cosine pulse are 0 at integer multiples of $T_{\text {sam }}$. Therefore, (10) reduces to

$$
r_{k}=\mathrm{e}^{j 2 \pi v\left(k T_{\mathrm{sam}}\right)} \sum_{n=0}^{L_{\mathrm{o}}-1} \gamma_{n} s\left(k T_{\text {sam }}-n T_{\text {sam }}\right)+w_{k}
$$

which is the system model used in [17], where the physical channel is represented by the commonly used tapped delay line model with equal tap spacing [21, p. 795]. Therefore, the channel model considered in this paper is more general than that in [17].

Remark 2: Note that since there are ten identical short training symbols transmitted at the beginning of the preamble, the frequency offset $v$ can be easily estimated by the ML frequency estimator [25] and may be compensated before frame synchronization. To simplify the development of the synchronization algorithms, $v=0$ is assumed in the following derivations. Performance of the developed algorithms in the presence of residual frequency offset after compensation will be assessed by simulations.

\section{B. Matrix Algebraic Formulations}

From (10), it is apparent that the received samples depend on $h\left(i T_{\text {sam }}\right)$ for $-\infty \leq i \leq \infty$. However, in practice, $h\left(i T_{\text {sam }}\right)$ will have significant values only for a finite range of $i$ since: 1) the path delays occur in a finite interval and 2) the value of $f(t)$ becomes very small when $|t|$ is large. An example of $\left|h\left(i T_{\mathrm{sam}}\right)\right|^{2}$ is shown in Fig. 2 for $L_{\mathrm{o}}=6$, where the first tap of the physical channel has zero delay, the other five taps have delay uniformly distributed over the interval 0-300 ns, $\gamma_{n}$ are independent and identically distributed (i.i.d.) zero-mean complex Gaussian random variables with variances following 
the multipath intensity profile $\phi(\tau) \sim \mathrm{e}^{-\tau / \tau_{\mathrm{rms}}}$, where $\tau_{\mathrm{rms}}=$ $100 \mathrm{~ns}, \varepsilon_{\mathrm{o}}$ is a random variable uniformly distributed in $[0,1)$, and $f(t)$ is given by (11), with $\beta=0.1$. It can be seen that $h\left(i T_{\text {sam }}\right)$ can be well represented by a sequence with finite length. Therefore, (10) can be approximated by (ignoring the frequency offset)

$$
r_{k} \cong \sum_{i=-L_{1}}^{L_{\mathrm{e}}-L_{1}-1} s\left(k T_{\mathrm{sam}}-i T_{\mathrm{sam}}\right) h\left(i T_{\mathrm{sam}}\right)+w_{k}
$$

where $L_{\mathrm{e}}$ and $L_{1}$ are the total number of taps and the number of taps for $t<0$ in the approximated equivalent channel, respectively. Note that the above approximation can be made arbitrarily accurate by using large-enough values of $L_{\mathrm{e}}$ and $L_{1}$.

Let $\mathbf{r}_{n}$ be a received-signal vector with $N$ received samples ${ }^{3}$

$$
\mathbf{r}_{n}=\left[\begin{array}{llll}
r_{n} & r_{n+1} & \cdots & r_{n+N-1}
\end{array}\right]^{\mathrm{T}}
$$

where $N=T_{b} / T_{\text {sam }}=16$ is the number of samples over the span of a short training symbol $b(t)$. Let $b_{n}=b\left(n T_{\text {sam }}\right)$ and $g_{n}=g\left(n T_{\text {sam }}\right)$ be the $n$th samples of the short training symbol and of the cyclic prefix, respectively. For $L_{\mathrm{e}}-L_{1} \leq n \leq$ $9 N-L_{1}, \mathbf{r}_{n}$ is given by

$$
\mathbf{r}_{n}=\sqrt{2 P} \mathbf{B}_{n+L_{1}}^{\left(L_{\mathrm{e}}\right)} \mathbf{h}_{\mathrm{o}}+\mathbf{w}_{n}
$$

where we have (18) and (19) shown at the bottom of the page, and $\mathbf{w}_{n}$ is a vector containing the noise samples with a covariance matrix $\sigma_{w}^{2} \mathbf{I}_{N}\left(\mathbf{I}_{N}\right.$ being the $N \times N$ identity matrix $)$. Similarly, the first received-signal vector for the cyclic prefix is given by

$$
\mathbf{r}_{n}=\sqrt{2 P} \mathbf{G}_{0}^{\left(L_{\mathrm{e}}\right)} \mathbf{h}_{\mathrm{o}}+\mathbf{w}_{n}, \quad \text { for } n=10 N-L_{1}
$$

where

$$
\mathbf{G}_{0}^{(L)} \triangleq\left[\begin{array}{ccccc}
g_{0} & b_{15} & b_{14} & \ldots & b_{16-L+1} \\
g_{1} & g_{0} & b_{15} & \ldots & b_{16-L+2} \\
g_{2} & g_{1} & g_{0} & \ldots & b_{16-L+3} \\
\vdots & \vdots & \vdots & \ddots & \vdots \\
g_{15} & g_{14} & g_{13} & \ldots & g_{16-L}
\end{array}\right]
$$

For notational simplicity, we write $\mathbf{B}_{n}^{(L)}$ and $\mathbf{G}_{0}^{(L)}$ as $\mathbf{B}_{n}$ and $\mathbf{G}_{0}$, respectively, in the subsequent derivations.

\footnotetext{
${ }^{3}$ Throughout this paper, the notations $(\cdot)^{*},(\cdot)^{\mathrm{T}},(\cdot)^{\mathrm{H}}$, and $\|\cdot\|$ stand for conjugate, transpose, conjugate transpose, and Euclidean norm, respectively.
}

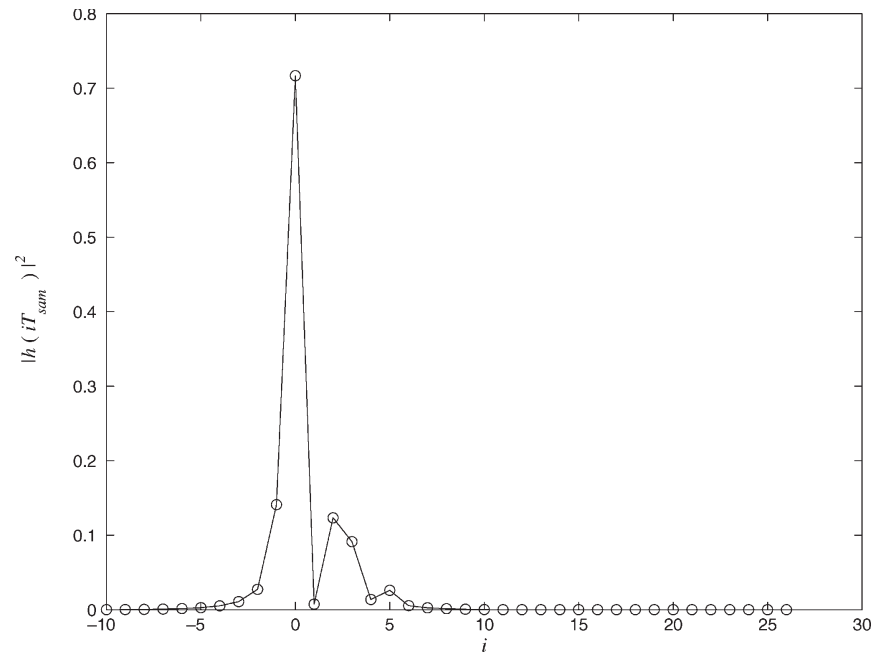

Fig. 2. Example of $\left|h\left(i T_{\mathrm{sam}}\right)\right|^{2}$ for $L_{\mathrm{o}}=6$, the first tap of the physical channel has zero delay, the other five taps have delays uniformly distributed over the interval 0-300 ns, $\alpha_{n}$ are i.i.d. zero-mean complex Gaussian random variables with variances following the multipath intensity profile $\phi(\tau) \sim \mathrm{e}^{-\tau / \tau_{\mathrm{rms}}}$, where $\tau_{\mathrm{rms}}=100 \mathrm{~ns}, \varepsilon_{\mathrm{o}}$ is a random variable uniformly distributed in $[0,1)$, and $f(t)$ is given by (11), with $\beta=0.1$.

Remark 3: The equivalent channel, which includes the effect of filtering and sampling offset in addition to the physical channel, is, in general, noncausal and has length longer than the span of the physical channel. For the channel example shown in Fig. 2, the path delays of the physical channel lie within $[0,300 \mathrm{~ns}]$, however, the equivalent channel has nonzero taps before $t=0$ and has length longer than $300 \mathrm{~ns}$ (although the "head" and "tail" of the equivalent channel are very small).

\section{Symbol-SYNCHRONIZATION-PERFORMANCE CRITERION}

Having established the system model, the next question is how to define the "beginning" of an OFDM symbol. For frequency flat fading channels, the starting position is obvious and well defined. For Rician fading channels, it is reasonable to define the symbol boundary with respect to the first path. However, in a Rayleigh multipath fading channel (e.g., the one shown in Fig. 2), the channel contains some small taps at the beginning and the starting position of the channel is not clear. It can be defined as the first nonzero tap of the channel, as the first tap with energy larger than a certain threshold, as the position of the strongest path or any other definition. Because of this, the symbol boundary of a received OFDM symbol is not well defined. Even if we choose one of the above definitions as the reference position, there is no guarantee that a

$$
\begin{aligned}
& \mathbf{B}_{n}^{(L)} \triangleq\left[\begin{array}{cccc}
b_{\bmod (n, 16)} & b_{\bmod (n-1,16)} & \cdots & b_{\bmod (n-L+1,16)} \\
b_{\bmod (n+1,16)} & b_{\bmod (n, 16)} & \cdots & b_{\bmod (n-L+2,16)} \\
\vdots & \vdots & \ddots & \vdots \\
b_{\bmod (n+15,16)} & b_{\bmod (n+14,16)} & \cdots & b_{\bmod (n-L+16,16)}
\end{array}\right] \\
& \mathbf{h}_{\mathrm{o}} \triangleq\left[h\left(-L_{1} T_{\mathrm{sam}}\right) \quad h\left(\left(-L_{1}+1\right) T_{\mathrm{sam}}\right) \quad \cdots \quad h\left(\left(L_{\mathrm{e}}-L_{1}+1\right) T_{\mathrm{sam}}\right)\right]^{\mathrm{T}}
\end{aligned}
$$




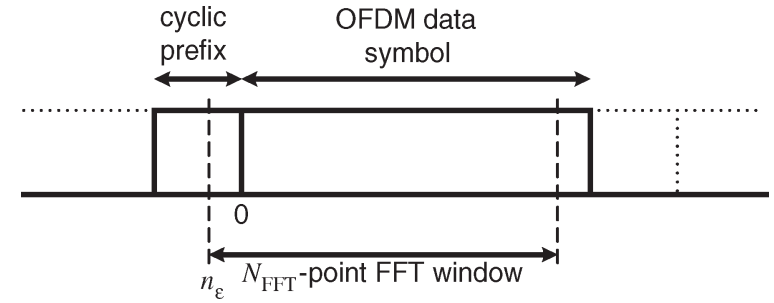

Fig. 3. OFDM symbol and FFT position.

certain synchronization algorithm giving estimates close to the reference position would provide good performance in OFDM systems. Moreover, in OFDM systems, due to the existence of cyclic prefix, some timing offset can be tolerated as long as the samples within the FFT window are influenced by only one transmitted OFDM symbol. Therefore, the criterion that the synchronization error has to be within certain limits of a fixed reference point is not an appropriate performance measure for OFDM systems in frequency-selective fading channels.

A more general and meaningful performance measure is the loss in system performance due to the synchronization error. With reference to Fig. 3, if the FFT window starts at position $n_{\varepsilon}$, the signal at the subcarrier $k$ after the FFT operation $z_{k}$ can be described as [27]

$$
z_{k}=\mathrm{e}^{j 2 \pi\left(\frac{k}{N_{\mathrm{FFT}}}\right) n_{\varepsilon}} \alpha\left(n_{\varepsilon}\right) a_{k} H_{k}+I_{k}+\eta_{k}
$$

where $a_{k}, H_{k}$, and $\eta_{k}$ are the data, channel transfer function, and noise sample at subcarrier $k$, respectively, $N_{\mathrm{FFT}}$ is the number of FFT points in the OFDM system, which is 64 in IEEE $802.11 \mathrm{a}, \alpha\left(n_{\varepsilon}\right)$ is the attenuation caused by the synchronization error, which can be well approximated by [27]

$$
\alpha\left(n_{\varepsilon}\right)=\sum_{i}\left|h\left(i T_{\mathrm{sam}}\right)\right|^{2} \frac{N_{\mathrm{FFT}}-\Delta \varepsilon_{i}}{N_{\mathrm{FFT}}}
$$

where

$$
\Delta \varepsilon_{i} \triangleq \begin{cases}n_{\varepsilon}-i, & n_{\varepsilon}>i \\ i-N-n_{\varepsilon}, & n_{\varepsilon}<-(N-i) \\ 0, & \text { otherwise }\end{cases}
$$

and $I_{k}$ is the ISI-plus-intercarrier-interference (ICI) term at subcarrier $k$ caused by the timing offset, which can be well approximated by Gaussian noise with power [27]

$$
\sigma_{\varepsilon}^{2}\left(n_{\varepsilon}\right)=\sum_{i}\left|h\left(i T_{\mathrm{sam}}\right)\right|^{2}\left(2 \frac{\Delta \varepsilon_{i}}{N_{\mathrm{FFT}}}-\left(\frac{\Delta \varepsilon_{i}}{N_{\mathrm{FFT}}}\right)^{2}\right) .
$$

For a particular channel realization, the signal-to-interferenceplus-noise ratio (SINR) is given by

$$
\operatorname{SINR}\left(n_{\varepsilon}\right)=\frac{\alpha^{2}\left(n_{\varepsilon}\right) \mathbb{E}\left[\left|a_{k} H_{k}\right|^{2}\right]}{\sigma_{\varepsilon}^{2}\left(n_{\varepsilon}\right)+\sigma_{w}^{2}} .
$$

Notice that for the special case that the equivalent channel length is smaller than the length of cyclic prefix (i.e., $L_{\mathrm{e}}<N$ ) and $L_{\mathrm{e}}-N \leq n_{\varepsilon} \leq 0$, then $\sigma_{\varepsilon}^{2}=0$, implying there is no ISI and ICI. On the other hand, if the length of the equivalent channel is larger than the length of the cyclic prefix, then $\sigma_{\varepsilon}^{2}>0$, therefore some ISI and ICI occur. In this case, the best we can do is to find a value of $n_{\varepsilon}$ that maximizes the SINR. Note that, due to (22), the SINR expression can be rewritten as

$$
\operatorname{SINR}\left(n_{\varepsilon}\right)=\frac{\mathbb{E}\left[\left|z_{k}\right|^{2}\right]-\sigma_{\varepsilon}^{2}\left(n_{\varepsilon}\right)-\sigma_{w}^{2}}{\sigma_{\varepsilon}^{2}\left(n_{\varepsilon}\right)+\sigma_{w}^{2}} .
$$

Since the sum of the average powers of the useful and the interference components in (22) is a constant [28], it follows that $\mathbb{E}\left[\left|z_{k}\right|^{2}\right]$ is also a constant. Therefore, maximizing SINR is equivalent to minimizing $\sigma_{\varepsilon}^{2}\left(n_{\varepsilon}\right)$, and the "ideal" symbol synchronizer should select $n_{\varepsilon}$ such that $\sigma_{\varepsilon}^{2}\left(n_{\varepsilon}\right)$ in (25) is minimized.

In practice, the ideal symbol synchronizer is not realizable since it requires a perfect knowledge of the channel realization. However, the ideal symbol synchronizer can serve as a reference to other practical synchronization algorithms. For a particular realization of channel, let $n_{\varepsilon}$ be the start of the FFT window estimated by a particular symbol-synchronization algorithm and $n_{\text {id }}$ be that from the ideal symbol synchronizer. Then, the loss of SINR, defined as the ratio of SINR obtained from the ideal symbol synchronizer to that from the nonideal symbol synchronizer, is given by

$$
\operatorname{SINR}_{\text {loss }}\left(n_{\varepsilon}\right) \triangleq \frac{\operatorname{SINR}_{\mathrm{id}}}{\operatorname{SINR}\left(n_{\varepsilon}\right)}=\frac{\alpha^{2}\left(n_{\mathrm{id}}\right)\left[\sigma_{\varepsilon}^{2}\left(n_{\varepsilon}\right)+\sigma_{w}^{2}\right]}{\alpha^{2}\left(n_{\varepsilon}\right)\left[\sigma_{\varepsilon}^{2}\left(n_{\mathrm{id}}\right)+\sigma_{w}^{2}\right]} .
$$

For a good symbol-synchronization algorithm, the loss in SINR with respect to the ideal synchronizer should be very small. Similar to [5], we define a synchronization failure as the event that the loss in SINR is greater than a tolerable system degradation, i.e.,

$$
P_{\mathrm{f}}(\Delta \gamma) \triangleq P\left(10 \log _{10}\left(\operatorname{SINR}_{\text {loss }}\right)>\Delta \gamma\right)
$$

where $P_{\mathrm{f}}(\Delta \gamma)$ is the probability of synchronization failure given that the tolerable system degradation (in decibels) is $\Delta \gamma$, and $P(\cdot)$ denotes the probability of an event.

Remark 1: Plugging (24) into (25), the power of the ISIplus-ICI term can be written as

$$
\begin{aligned}
\sigma_{\varepsilon}^{2}\left(n_{\varepsilon}\right)= & \sum_{i=-\infty}^{n_{\varepsilon}-1}\left|h\left(i T_{\mathrm{sam}}\right)\right|^{2}\left(2 \frac{n_{\varepsilon}-i}{N_{\mathrm{FFT}}}-\left(\frac{n_{\varepsilon}-i}{N_{\mathrm{FFT}}}\right)^{2}\right) \\
& +\sum_{i=n_{\varepsilon}+N+1}^{\infty}\left|h\left(i T_{\mathrm{sam}}\right)\right|^{2} \\
& \times\left(2 \frac{i-N-n_{\varepsilon}}{N_{\mathrm{FFT}}}-\left(\frac{i-N-n_{\varepsilon}}{N_{\mathrm{FFT}}}\right)^{2}\right) .
\end{aligned}
$$

The meaning of minimizing (30) is to find a starting position $n_{\varepsilon}$ for a rectangular window of length $N+1$ points such that the weighted sum of the energy of the channel taps outside this rectangular window is minimized. It is mentioned in [28] that, for simplicity, minimizing (30) can be replaced by minimizing

$$
\tilde{\sigma}_{\varepsilon}^{2}\left(n_{\varepsilon}\right)=\sum_{i=-\infty}^{n_{\varepsilon}-1}\left|h\left(i T_{\mathrm{sam}}\right)\right|^{2}+\sum_{i=n_{\varepsilon}+N+1}^{\infty}\left|h\left(i T_{\mathrm{sam}}\right)\right|^{2} .
$$


Further notice that minimizing (31) is equivalent to maximizing

$$
\sum_{i=n_{\varepsilon}}^{n_{\varepsilon}+N}\left|h\left(i T_{\mathrm{sam}}\right)\right|^{2}
$$

which is the optimal timing criterion proposed in [2, pp. 90-92].

\section{Proposed Symbol-Synchronization Algorithm}

For the packet structure shown in Fig. 1, determining the FFT-window position for the OFDM data symbols actually involves two major steps. The first one is to identify a reference position (e.g., the transition form the short training symbol to the cyclic prefix of the long training symbol in the middle of the preamble) such that all the subsequent transitions can be predicted. We refer to this step as frame synchronization. The second step is to locate the FFT-window position within an OFDM symbol such that the ISI plus ICI introduced is minimized, which we refer to as symbol synchronization. Notice that in some cases, frame synchronization and symbol synchronization are the same process. For example, if the channel is frequency flat or the multipath channel is causal and with total length smaller than the cyclic prefix of the OFDM symbols, then the optimum position for the FFT window is at $n_{\varepsilon}=0$ [28], and symbol synchronization follows naturally and immediately once frame synchronization is achieved. However, for the channel shown in Fig. 2, which is noncausal and the total length may be larger than the cyclic prefix of the OFDM symbols, symbol synchronization is essential.

\section{A. Frame Synchronization}

Suppose that the arrival of the preamble can be identified by detecting the received-signal energy (e.g., using the methods in [8] or [12]), the problem of detecting the transition between the short training symbols and the cyclic prefix of the long training symbols can be decomposed into two subproblems. Let $\mathbf{r}_{n_{1}}$ be a received-signal vector within the short training interval. Since $\mathbf{r}_{n_{1}}$ may not align with the beginning of a short training symbol, the first subproblem is to determine the current time offset with respect to the last short training symbol, and hence, predict the starting time of the next (expected) short training symbol. Suppose this starting time is denoted by the time index $n_{2}$. After $n_{2}$ is obtained, the second subproblem is to examine the incoming vectors $\mathbf{r}_{n_{2}+q N}, q=0,1,2, \ldots, 10$, and to determine if they belong to a short training symbol or a cyclic prefix of the long training symbol. The transition point is declared at the time instant that the first $\mathbf{r}_{n_{2}+q N}$ belongs to the cyclic prefix.

1) First Stage: Assume that the received-signal vector $\mathbf{r}_{n_{1}}$ is $i_{\mathrm{o}}$ samples $\left(i_{\mathrm{o}} \in\{0,1, \ldots, 15\}\right)$ offset from the beginning of the current short training symbol. The probability density function (pdf) of the received-signal vector $\mathbf{r}_{n_{1}}$ is

$$
p\left(\mathbf{r}_{n_{1}} ; \boldsymbol{\theta}, i, L\right)=\frac{1}{\pi^{N} \sigma^{2 N}} \exp \left\{-\frac{1}{\sigma^{2}}\left\|\mathbf{r}_{n_{1}}-\mathbf{B}_{i} \sqrt{2 P} \mathbf{h}\right\|^{2}\right\}
$$

where $\boldsymbol{\theta}^{4} \triangleq\left[\Re\left(\sqrt{2 P} \mathbf{h}^{\mathrm{T}}\right) \quad \Im\left(\sqrt{2 P} \mathbf{h}^{\mathrm{T}}\right) \sigma^{2}\right]^{\mathrm{T}}$, with $\mathbf{h}, \sigma^{2}$, and $L$ being the trial values of $\mathbf{h}_{\mathrm{o}}, \sigma_{w}^{2}$, and $L_{\mathrm{e}}$, respectively. Note that $\mathbf{B}_{i}$ depends on $i$ and $L$ [see (18)]. It is not possible to jointly estimate $\mathbf{h}_{\mathrm{o}}, \sigma_{w}^{2}$, $i_{\mathrm{o}}$, and $L_{\mathrm{e}}$ by directly maximizing $p\left(\mathbf{r}_{n_{1}} ; \boldsymbol{\theta}, i, L\right)$ since (apart from the fact that the implementation complexity would be extremely high) the largest possible $L$ is always chosen [23, p. 223]. One criterion that gets around this problem is the generalized ML rule [23, p. 223], in which we maximize

$$
\Psi\left(\mathbf{r}_{n_{1}} ; i, L\right)=\ln p\left(\mathbf{r}_{n_{1}} ; \hat{\boldsymbol{\theta}}, i, L\right)-\frac{1}{2} \ln \operatorname{det}(\mathbf{I}(\hat{\boldsymbol{\theta}} \mid i, L))
$$

where the second term is a penalty term that becomes more negative as $L$ increases. In the above expression, $\hat{\boldsymbol{\theta}}$ is the ML estimate of $\boldsymbol{\theta}$ (given $i$ and $L$ ) with elements given by [22, p. 186] [23, p. 222]

$$
\begin{aligned}
\widehat{\sqrt{2 P} \mathbf{h}} & =\left(\mathbf{B}_{i}^{\mathrm{H}} \mathbf{B}_{i}\right)^{-1} \mathbf{B}_{i}^{\mathrm{H}} \mathbf{r}_{n_{1}} \\
\hat{\sigma}^{2} & =\frac{1}{N}\left\|\mathbf{r}_{n_{1}}-\mathbf{B}_{i}\left(\mathbf{B}_{i}^{\mathrm{H}} \mathbf{B}_{i}\right)^{-1} \mathbf{B}_{i}^{\mathrm{H}} \mathbf{r}_{n_{1}}\right\|^{2}
\end{aligned}
$$

and $\mathbf{I}(\boldsymbol{\theta} \mid i, L)$ is the Fisher information matrix of $\boldsymbol{\theta}$ (given $i$ and $L$ ) with elements given by [22, p. 525]

$$
\begin{aligned}
{[\mathbf{I}(\boldsymbol{\theta} \mid i, L)]_{m n}=} & \frac{1}{\sigma^{4}}\left[\frac{\partial \sigma^{2}}{\partial \theta_{m}} \frac{\partial \sigma_{2}}{\partial \theta_{n}}\right] \\
& +\frac{2}{\sigma^{2}} \Re\left[\frac{\partial\left(\mathbf{B}_{i} \sqrt{2 P} \mathbf{h}\right)^{\mathrm{H}}}{\partial \theta_{m}} \frac{\partial\left(\mathbf{B}_{i} \sqrt{2 P} \mathbf{h}\right)}{\partial \theta_{n}}\right]
\end{aligned}
$$

where $\theta_{m}$ is the $m$ th element of $\boldsymbol{\theta}$.

As discussed in Section III, since the starting position of a frame is not well defined in multipath Rayleigh fading channels, it is necessary to clarify what is the meaning of estimated offset by maximizing (34). Let $\{\hat{i}, \hat{L}\}$ be the set of values that maximize (34), then $\hat{i}$ is the number of offset samples from the beginning of the current short training symbol conditioned that the number of paths of the channel is $\hat{L}$ and the offset is with respect to the first estimated path. Notice that the generalized ML criterion (34) tends to ignore the channel paths of small energy, therefore, $\hat{L}<L_{\mathrm{e}}$. For example, in the channel shown in Fig. 2, the estimated channel length from the generalized ML criterion is $\hat{L}=8(-2 \leq i \leq 5)$ at $\mathrm{SNR}=30 \mathrm{~dB}$.

It is proved in Appendix that, the generalized ML rule (34) can be simplified to

$$
\begin{aligned}
& \Psi_{1}\left(\mathbf{r}_{n_{1}} ; i, L\right)=(-N+L+1) \\
& \quad \times \ln \left\|\mathbf{r}_{n_{1}}-\mathbf{B}_{i}\left(\mathbf{B}_{0}^{\mathrm{H}} \mathbf{B}_{0}\right)^{-1} \mathbf{B}_{i}^{\mathrm{H}} \mathbf{r}_{n_{1}}\right\|^{2}-\xi(L)
\end{aligned}
$$

where $\xi(L) \triangleq L \ln 2+\ln \left(\operatorname{det}\left(\mathbf{B}_{0}^{\mathrm{H}} \mathbf{B}_{0}\right)\right)$ is a function of $L$ only and can be precomputed and stored in a look-up table to reduce the real-time computational complexity. The first-stage synchronization algorithm, which jointly estimates the effective

\footnotetext{
${ }^{4}$ Notations $\Re(\cdot)$ and $\Im(\cdot)$ denote the real part and imaginary part, respectively.
} 
channel order $L_{\mathrm{e}}$ and the delay $i_{\mathrm{o}}$ from the received-signal vector $\mathbf{r}_{n_{1}}$, becomes

$$
\hat{i}, \hat{L}=\arg \max _{\substack{i \in\{0,1, \ldots, 15\}, L \in\left\{1,2, \ldots, L_{\max }\right\}}} \Psi_{1}\left(\mathbf{r}_{n_{1}} ; i, L\right)
$$

where $L_{\max }$ is the maximum possible value of the channel order. The starting position of the next expected short training symbol is then given by $n_{2}=n_{1}+16-\hat{i}$.

2) Second Stage: The second step is to determine the smallest value of $q$ such that $\mathbf{r}_{n_{2}+q N}$ belongs to the cyclic prefix. This problem can be handled by the Neyman-Pearson (NP) detection approach [23, Ch. 3]. Let $H_{g}$ and $H_{b}$ be the hypotheses that $\mathbf{r}_{n_{2}+q N}$ belongs to the cyclic prefix and the short training symbol, respectively. In each test, the probability that the received-signal vector belongs to the short training symbol is the same as the probability that it belongs to the cyclic prefix. It follows that $n_{2}+q N$ is the point of transition if the condition

$$
p\left(\mathbf{r}_{n_{2}+q N} \mid H_{g}\right)>p\left(\mathbf{r}_{n_{2}+q N} \mid H_{b}\right)
$$

occurs for the first time, where

$$
\begin{aligned}
& p\left(\mathbf{r}_{n_{2}+q N} \mid H_{g}\right) \\
& \left.\quad=\frac{1}{\pi^{N} \sigma^{2 N}} \exp \left\{-\frac{1}{\sigma^{2}}\left\|\mathbf{r}_{n_{2}+q N}-\mathbf{G}_{0} \sqrt{2 P} \mathbf{h}\right\|^{2}\right\}\right]_{L=\hat{L}} \\
& p\left(\mathbf{r}_{n_{2}+q N} \mid H_{b}\right) \\
& \left.\quad=\frac{1}{\pi^{N} \sigma^{2 N}} \exp \left\{-\frac{1}{\sigma^{2}}\left\|\mathbf{r}_{n_{2}+q N}-\mathbf{B}_{0} \sqrt{2 P} \mathbf{h}\right\|^{2}\right\}\right]_{L=\hat{L}} .
\end{aligned}
$$

Taking logarithm on both sides of (40), and putting $\widehat{\sqrt{2 P} \mathbf{h}}=\left(\mathbf{G}_{0}^{\mathrm{H}} \mathbf{G}_{0}\right)^{-1} \mathbf{G}_{0}^{\mathrm{H}} \mathbf{r}_{n_{2}+q N}$ into (41) and $\widehat{\sqrt{2 P} \mathbf{h}}=$ $\left(\mathbf{B}_{0}^{\mathrm{H}} \mathbf{B}_{0}\right)^{-1} \mathbf{B}_{0}^{\mathrm{H}} \mathbf{r}_{n_{2}+q N}$ into (42), we find that $n_{2}+q N$ is the point of transition if

$$
\Psi_{2}\left(\mathbf{r}_{n_{2}+q N} \mid H_{g}\right)>\Psi_{2}\left(\mathbf{r}_{n_{2}+q N} \mid H_{b}\right)
$$

where

$$
\begin{aligned}
& \left.\Psi_{2}\left(\mathbf{r}_{n_{2}+q N} \mid H_{g}\right)=\mathbf{r}_{n_{2}+q N}^{\mathrm{H}} \mathbf{G}_{0}\left(\mathbf{G}_{0}^{\mathrm{H}} \mathbf{G}_{0}\right)^{-1} \mathbf{G}_{0}^{\mathrm{H}} \mathbf{r}_{n_{2}+q N}\right]_{L=\hat{L}} \\
& \left.\Psi_{2}\left(\mathbf{r}_{n_{2}+q N} \mid H_{b}\right)=\mathbf{r}_{n_{2}+q N}^{\mathrm{H}} \mathbf{B}_{0}\left(\mathbf{B}_{0}^{\mathrm{H}} \mathbf{B}_{0}\right)^{-1} \mathbf{B}_{0}^{\mathrm{H}} \mathbf{r}_{n_{2}+q N}\right]_{L=\hat{L}} .
\end{aligned}
$$

\section{B. Position of FFT Window}

After the transition between short training symbols and the cyclic prefix of the long training symbols is detected, we can predict that the beginning of the first data-carrying OFDM symbol (the OFDM symbol for the signal field) is $n_{3} \triangleq n_{2}+\hat{q} N+$ $(32+2 \times 64)$, where $32+2 \times 64$ is the number of samples for the long training symbols. If the equivalent channel has exactly $\hat{L}$ paths, then the allowable range for the starting point of the
FFT window is $\left\{n_{3}+\hat{L}, \ldots, n_{3}+N\right\}$. However, in reality, it is expected that there are some channel taps with small energy preceding and following the $\hat{L}$ paths. With the observation that the "head" and "tail" of the equivalent channel have energy die down more or less at the same rate, it is reasonable to set the start of the FFT window at

$$
n_{4} \triangleq n_{3}+\hat{L}+\left\lfloor\frac{(N-\hat{L})}{2}\right\rfloor .
$$

For subsequent OFDM symbols, the starting points of the FFT window are then $n_{4}+\ell\left(N+N_{\mathrm{FFT}}\right)$ where $\ell$ is a positive integer.

\section{Summary and Remarks}

The proposed symbol-synchronization procedure is summarized as follows.

Step 1) Take a received-signal vector $\mathbf{r}_{n_{1}}$ of length $N$ at any starting position $n_{1}$ after the signal is detected. Find estimated values of $i$ and $L$ such that $\Psi_{1}\left(\mathbf{r}_{n_{1}} ; i, L\right)$ is maximized over $i \in\{0,1, \ldots, 15\}$ and $L \in\left\{1,2, \ldots, L_{\max }\right\}$, where $\Psi_{1}\left(\mathbf{r}_{n_{1}} ; i, L\right)$ is given by (38). Calculate $n_{2}=n_{1}+16-\hat{i}$.

Step 2) Examine $\mathbf{r}_{n_{2}+q N}, \quad q=0,1, \ldots, 10$. Find the smallest value of $q$ such that $\Psi_{2}\left(\mathbf{r}_{n_{2}+q N} \mid H_{g}\right)>$ $\Psi_{2}\left(\mathbf{r}_{n_{2}+q N} \mid H_{b}\right)$, where $\Psi_{2}\left(\mathbf{r}_{n_{2}+q N} \mid H_{g}\right)$ and $\Psi_{2}\left(\mathbf{r}_{n_{2}+q N} \mid H_{b}\right)$ are given by (44) and (45), respectively. Declare $n_{2}+\hat{q} N$ as the time of transition.

Step 3) The FFT window for the first OFDM symbol should start at $n_{4} \triangleq n_{2}+\hat{q} N+(32+2 \times 64)+$ $\hat{L}+\lfloor(N-\hat{L}) / 2\rfloor$. For subsequent OFDM symbols, the starting points of the FFT window are then $n_{4}+\ell\left(N+N_{\mathrm{FFT}}\right)$, where $\ell$ is a positive integer.

We make the following remarks.

1) A knowledge of $n_{1}$ is, in general, not available at the receiver, so that the best knowledge that can be obtained after accomplishing Step 1) is the difference $n_{2}-n_{1}$. Despite this, it is sufficient for the receiver to locate the next expected short training symbol.

2) In the practical implementation of Step 2), the receiver need not examine the entire sequence of $\mathbf{r}_{n 2+q N}$. The time of transition can be declared right after the condition for the test is met. The rest of the $\mathbf{r}_{n 2+q N}$ vectors can be ignored.

3) For (35) to hold, it is required that $N>L$ and $\mathbf{B}_{i}$ be of full rank (i.e., $\operatorname{rank}\left(\mathbf{B}_{i}\right)=L$ ) [22, p.186]. Numerical calculation shows that the maximum value of $L$ that makes $\mathbf{B}_{i}$ full rank is $L_{\max }=12$, which corresponds to a time duration of $600 \mathrm{~ns}$. As WLANs are mainly used inside buildings, and measurements show that the maximum delay spreads of physical channels in different indoor environments are only about $300 \mathrm{~ns}$ [2, pp. 18-19], together with the fact that the generalized ML criterion treats the channel taps with small energies as noise, the proposed synchronizer can handle most practical situations. 
4) It is interesting to note that the form of the generalized ML rule in (38) is similar to the GAIC used in [17]. However, there are several differences between the algorithm proposed here and the one in [17]. First, our proposed algorithm uses the short training symbols and the cyclic prefix of the long training symbol to achieve frame synchronization whereas the algorithm in [17] uses only the long training symbol. Second, the observation-vector length used in the proposed algorithm is 16 whereas it is 64 for the one in [17]. Third, our proposed algorithm is developed based on a time-domain approach while a frequency-domain analysis is employed in [17]. Lastly, our proposed algorithm considers a more general channel model than the algorithm in [17] (see Remark 1 of Section II-A). Performance and complexity comparisons between the proposed algorithm and the technique of [17] are provided in the next section.

5) If $L$ is perfectly known, the metric in the first stage reduces to the same form as the metric in the second stage. Furthermore, in this case, the proposed first-stage detection algorithm coincides with the general frame synchronizer for packet-based transmissions in frequencyselective fading channels proposed in [18]-[20] (with the frequency offset equal to 0 ).

\section{Simulation Results and Discussions}

\section{A. Simulation Conditions}

Simulations are run in order to investigate the synchronization performance of the proposed algorithm. The received samples are generated according to (15) with $L_{1}=15$ and $L_{\mathrm{e}}=36$ so that the range of index $i$ in $(15)$ is $\{-15, \ldots, 20\}$. This enables the equivalent channel to be accurately represented (see Fig. 2). The channel gains $\gamma_{n}, n=0,1, \ldots, L_{\mathrm{o}}-1$, were modeled as mutually independent circularly symmetric zeromean complex Gaussian random variables (i.e., Rayleigh fading was considered). The number of physical paths is $L_{\mathrm{o}}=6$. The channel dispersion was modeled by an exponential function with $\phi(\tau) \sim \mathrm{e}^{-\tau / \tau_{\text {rms }}}$, where $\tau_{\text {rms }}=100$ ns. The combined transmit and receive filter $f(t)$ is given by a raised-cosine filter (11) with $\beta=0.1$. Two different models for the arrival time of the channel paths are considered. The first one (referred to as the channel I) assumes that the path delays are sample spaced (i.e., $\left.\tau_{n}=n T_{\text {sam }}, n=0,1, \ldots, 5\right)$ and there is no sampling phase offset (i.e., $\varepsilon_{\mathrm{o}}=0$ ). The second one (referred to as the channel II) is more realistic and assumes the first tap has zero delay, the other five taps present delays uniformly distributed over the interval [0-300 ns], and the fractional timing delay $\varepsilon_{\mathrm{o}}$ is treated as a uniform random variable over $[0,1)$. Note that from the measurements performed in indoor channels [2, pp. 18-19], the parameters of channel II basically represent the worst case channel in indoor environments. The channel is fixed during each packet but independent from one packet to another.

As a working assumption, we follow a suggestion of the standard [1] that the last three short training symbols are used for frame synchronization, although in practice it varies from
TABLE I

Probability of SyNChronization FAILURE FOR THE PRoposed ALGORITHM IN CHANNEL II AT SNR $=25 \mathrm{~dB}$ AND DIFFERENT FREQUENCY OFFSETS

\begin{tabular}{|c|c|c|}
\hline$v$ & $P_{f}(0.5 \mathrm{~dB})$ & $P_{f}(1 \mathrm{~dB})$ \\
\hline$-240 \mathrm{kHz}$ & $3.1 \times 10^{-3}$ & $1 \times 10^{-4}$ \\
$-180 \mathrm{kHz}$ & $3.2 \times 10^{-3}$ & $2 \times 10^{-4}$ \\
$-120 \mathrm{kHz}$ & $2.9 \times 10^{-3}$ & $1 \times 10^{-4}$ \\
$-60 \mathrm{kHz}$ & $2.3 \times 10^{-3}$ & $1 \times 10^{-4}$ \\
0 & $3.2 \times 10^{-3}$ & 0 \\
$60 \mathrm{kHz}$ & $2.3 \times 10^{-3}$ & $1 \times 10^{-4}$ \\
$120 \mathrm{kHz}$ & $3.5 \times 10^{-3}$ & 0 \\
$180 \mathrm{kHz}$ & $2.6 \times 10^{-3}$ & $1 \times 10^{-4}$ \\
$240 \mathrm{kHz}$ & $4.2 \times 10^{-3}$ & $1 \times 10^{-4}$ \\
\hline
\end{tabular}

one situation to another. Therefore, $n_{1}$ was treated as a uniform random variable over $[5 N+1,6 N]$ in the simulation, and a value of $n_{1}$ was randomly generated in each run. For each simulation run, the loss of SINR is calculated using (28), where the ideal symbol synchronizer selects a starting point for the FFT window such that (31) is minimized [simulation results not shown in this paper show that minimizing (30) or (31) give the same results]. The noise samples are i.i.d. zero-mean complex Gaussian random variable. The SNR was defined as

$$
\mathrm{SNR} \triangleq \frac{\frac{1}{2} \mathbb{E}\left[|r(t)-w(t)|^{2}\right]}{\frac{1}{2} \mathbb{E}\left[|w(t)|^{2}\right]}=\frac{2 P}{\sigma_{w}^{2}}
$$

Each simulation point is obtained by averaging over $10^{4}$ Monte Carlo runs.

\section{B. Effect of Frequency Offset}

We first examine the effect of nonzero frequency offset. It is required that the deviation of the transmitted-signal center frequency is within $\pm 20 \mathrm{ppm}$ [1]. Assume that the receiver oscillator also has a frequency uncertainty of $\pm 20 \mathrm{ppm}$. Since the highest operating frequency of the WLAN is $5.8 \mathrm{GHz}$ [1], the worst case frequency offset in the receiver is $\pm 232 \mathrm{kHz}$. Table I lists the probability of synchronization failure, $P_{\mathrm{f}}(0.5 \mathrm{~dB})$ and $P_{\mathrm{f}}(1 \mathrm{~dB})$, for the proposed algorithm in channel II against different frequency offsets, under the condition of SNR $=25 \mathrm{~dB}$. The frequency offset is estimated as $\hat{v}=\arg \left\{\mathbf{r}_{n}^{\mathrm{H}} \mathbf{r}_{n+N}\right\} / 2 \pi N T_{\text {sam }}$ [25] for any $L_{\mathrm{e}}-L_{1} \leq n<n_{1}$ and compensated before frame synchronization. For the case of $v=0$, no frequency-offset estimation is performed and it serves as a reference. The results indicate that the presence of frequency offset does not have significant effect on the probability of synchronization failure. In generating the simulation results for the rest of this paper, we set $v=0$.

\section{Performances and Comparisons With Other Algorithms}

In this section, the performance of the proposed algorithm will be compared to an existing symbol-synchronization algorithm for IEEE 802.11a WLANs [17] and three other 
algorithms for general OFDM symbol synchronization [6], [12], [14]. The algorithms in [6], [12], and [14] are selected for comparison since they represent different types of techniques commonly used in symbol synchronization of OFDM systems. The algorithm in [6] represents a class of techniques that exploit the periodic structure of the received signal and requires no knowledge of the preamble pattern (autocorrelation technique); the algorithm in [12] represents a class of techniques that correlate the received signal with the known training signal (cross-correlation technique) and the algorithm in [14] stands for the technique that makes use of both the knowledge of transmitted preamble and the periodic structure (double-correlation technique).

The algorithm based on GAIC [17] is designed to detect the transition between $g(t)$ and $c(t)$ in the preamble. Due to the fact that the GAIC algorithm also provides an estimate of the channel length, the FFT-window starting position for the first OFDM data symbol can be calculated in a similar way to the proposed algorithm [see (46)]. That is, the FFT window starts at $\hat{n}_{\mathrm{GAIC}}+2 \times 64+\hat{L}_{\mathrm{GAIC}}+\left\lfloor\left(N-\hat{L}_{\mathrm{GAIC}}\right) / 2\right\rfloor$ where $\hat{n}_{\mathrm{GAIC}}$ and $\hat{L}_{\mathrm{GAIC}}$ are the timing estimate and channellength estimate from the GAIC algorithm, respectively. For the algorithms in [6], [12], and [14], since they are not originally designed for the IEEE 802.11a standard, they have to be slightly modified. Let $\overline{\mathbf{r}}_{n} \triangleq\left[\begin{array}{llll}r_{n} r_{n+1} & \cdots & r_{n+63}\end{array}\right]^{\mathrm{T}}$ and $\tilde{\mathbf{r}}_{n} \triangleq\left[\begin{array}{llll}r_{n} & r_{n+1} & \ldots & r_{n+95}\end{array}\right]^{\mathrm{T}}$ be the received-signal vectors of lengths 64 and 96, respectively, [an overbar or a tilde is added to distinguish them from the received-signal vector $\mathbf{r}_{n}$ of length 16 defined in (16)]. Furthermore, let

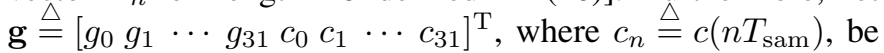
the known training sequence of length 64 , starting from the first sample of the cyclic prefix $g(t)$. Then, the modifications are as follows.

1) For the autocorrelation technique, the repetition structure in the long training symbols is being exploited. The transition between $b(t)$ and $g(t)$ is estimated by [6]

$$
\hat{n}_{\mathrm{AC}}=\arg \max _{n}\left\{\left|\tilde{\mathbf{r}}_{n}^{\mathrm{H}} \tilde{\mathbf{r}}_{n+64}\right|-\frac{\rho_{1}\left(\left\|\tilde{\mathbf{r}}_{n}\right\|^{2}+\left\|\tilde{\mathbf{r}}_{n+64}\right\|^{2}\right)}{2}\right\}
$$

where $\rho_{1} \triangleq \mathrm{SNR} /(1+\mathrm{SNR})$. Due to the structure of the long training symbols, an observation length of 96 is needed, otherwise, the cost function inside the max operation would have a plateau, leading to uncertainty for the start of the frame. Notice that similar algorithms have been proposed in [4], [5], and [7]. Once the transition between $b(t)$ and $g(t)$ is identified, the FFT-window starting position for the first OFDM data symbol would be $\hat{n}_{\mathrm{AC}}+(32+2 \times 64)+16-\lambda$, where $\lambda$ is the preadvancement to account for the mean shift of the estimated timing position caused by the channel dispersion [29]. As there is no channel-length information, the value of preadvancement $\lambda$ is chosen based on the following intuitive argument. Since the length of the cyclic prefix for the OFDM symbols is $800 \mathrm{~ns}$, and from the channel measurements, the maximum delay spread of the indoor physical channel is smaller than $300 \mathrm{~ns}$ [2, pp. 18-19], therefore, there will be about $500 \mathrm{~ns}$ of cyclic prefix that contains very small amount of ISI and it is safest to start the FFT window in the middle of this 500 -ns region. That is, $\lambda=500 \mathrm{~ns} / 2 T_{\text {sam }}=5$.

2) Define $Q_{n} \triangleq \mathbf{g}^{\mathrm{H}} \overline{\mathbf{r}}_{n}$ as the correlation between the received vector and the known training-sequence vector. Since the periodic autocorrelation property of vector $\mathrm{g}$ resembles that of a pseudonoise (PN) sequence (this can easily be shown by numerical computations), correlation peaks are expected if the received vector starts near the transition between $b(t)$ and $g(t)$. The cross-correlationbased algorithm in [12] is used to detect this correlation peak and can be stated as first finding the smallest value of $n$ such that $\left|Q_{n}+Q_{n+1}\right|^{2}-\rho_{2}\|\mathbf{g}\|^{2}\left\|\overline{\mathbf{r}}_{n}\right\|^{2}>0$, then, the frame position is given by

$$
\hat{n}_{\mathrm{CC}}=\arg \max _{n}\left\{\left|Q_{n}\right|,\left|Q_{n+1}\right|\right\}
$$

where $\rho_{2}=0.8$, as suggested in [13]. Similar to the autocorrelation algorithm, the FFT-window starting position for the first OFDM data symbol would be $\hat{n}_{\mathrm{CC}}+(32+$ $2 \times 64)+16-\lambda$.

3) For the double-correlation algorithm in [14], $Q_{n}$ is first generated and then the conjugate of the correlation outputs 64 samples later (i.e., $Q_{n+64}^{*}$ ) is multiplied with $Q_{n}$. According to Tufvesson et al. [14], the correlation peaks of the product $\left|Q_{n} Q_{n+64}^{*}\right|$ approximately correspond to the channel tap power for each delay. Therefore, based on the same rationale as the ideal synchronizer [see (32)], a sum of $\left|Q_{n} Q_{n+64}^{*}\right|$ over a rectangular window of length $N+1$ should be used to locate the correct timing. Mathematically, it can be stated as

$$
\hat{n}_{\mathrm{DC}}=\arg \max _{n}\left\{\sum_{i=n}^{n+N}\left|Q_{i} Q_{i+64}^{*}\right|\right\} .
$$

Then, the FFT-window starting position for the first OFDM data symbol is given by $\hat{n}_{\mathrm{DC}}+(32+2 \times 64)+$ 16 . No preadvancement is needed since the estimator (50) introduces the preadvancement implicitly.

Let us first consider the performances of different algorithms in channel I. Fig. 4 plots the distributions of the estimated FFT-window positions at $\mathrm{SNR}=30 \mathrm{~dB}$ with respect to $n_{\varepsilon}=0$ (reference to Fig. 3). Notice that for channel I, as long as the FFT window starts in the interval $n_{\varepsilon} \in\{-10, \ldots, 0\}$, there is no performance penalty. From Fig. 4, it can be seen that the proposed algorithm and the algorithm based on GAIC have very high estimation accuracies and all the estimated positions lie within the ISI-free region, while the other three correlationbased algorithms have larger estimation variances and present some estimated positions outside the ISI-free region (for the algorithm based on cross correlation [12], since the threshold $\rho_{2}$ is not optimal in frequency-selective fading channels, about $10 \%$ of estimated positions lie outside the display of this figure). One may argue that the performance of the doublecorrelation algorithm can be improved if all the estimates are 


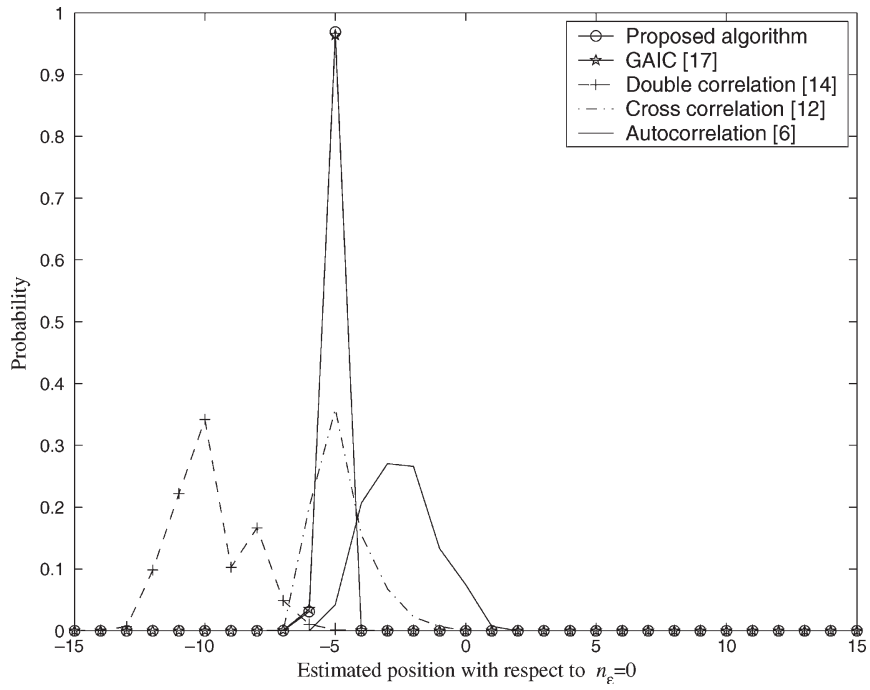

Fig. 4. Distributions of the detected FFT-window starting position for the proposed algorithm and algorithms in [6], [12], [14], and [17] with respect to the ideal FFT-window starting position in channel I.

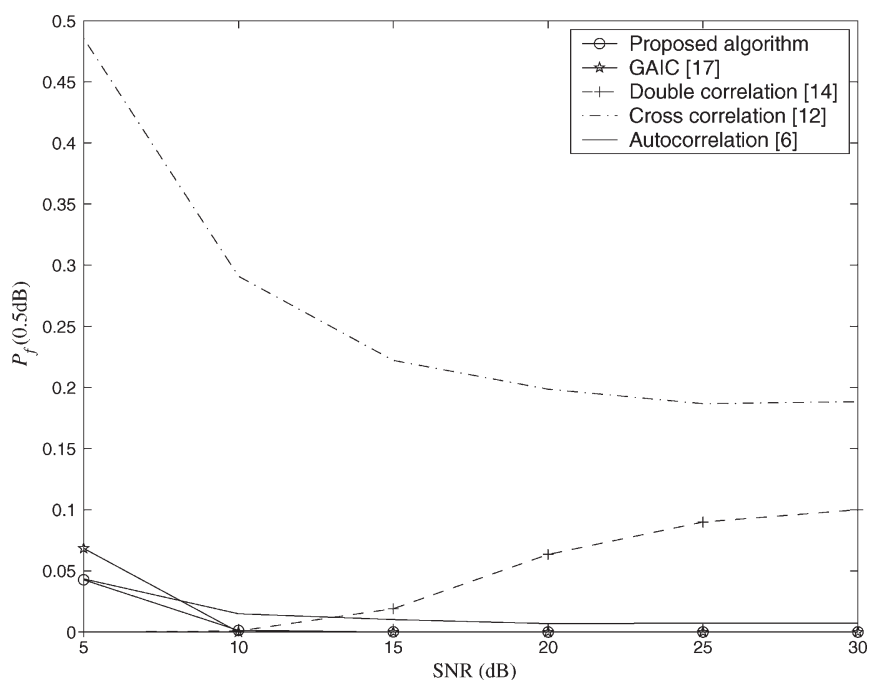

Fig. 5. $P_{\mathrm{f}}(0.5 \mathrm{~dB})$ for the proposed algorithm and algorithms in [6], [12], [14], and [17] as a function of SNR in channel I.

shifted by five samples to the right (similarly, the results of the autocorrelation algorithm can be corrected by shifting about three samples to the left). However, this cannot be done in practice since the amount of mean shifts are not known in reality. Notice that the mean shifts deduced from the simulated distributions are only available if: 1) a lot of trials were run and 2) the optimal frame position with respect to the estimated position is known. Unfortunately, these two conditions cannot be met in practice. Furthermore, the amount of mean shifts would highly depend on the channel characteristics (delay spread and the number of physical paths), which ultimately depend on the operating environment. The amount of mean shift suitable for one environment may not be suitable for another.

Fig. 5 plots the probability of synchronization failure $P_{\mathrm{f}}(0.5 \mathrm{~dB})$ as a function of SNR. It can be observed that the proposed algorithm and the algorithm based on GAIC have zero probability of synchronization failure for SNR $\geq 10 \mathrm{~dB}$.

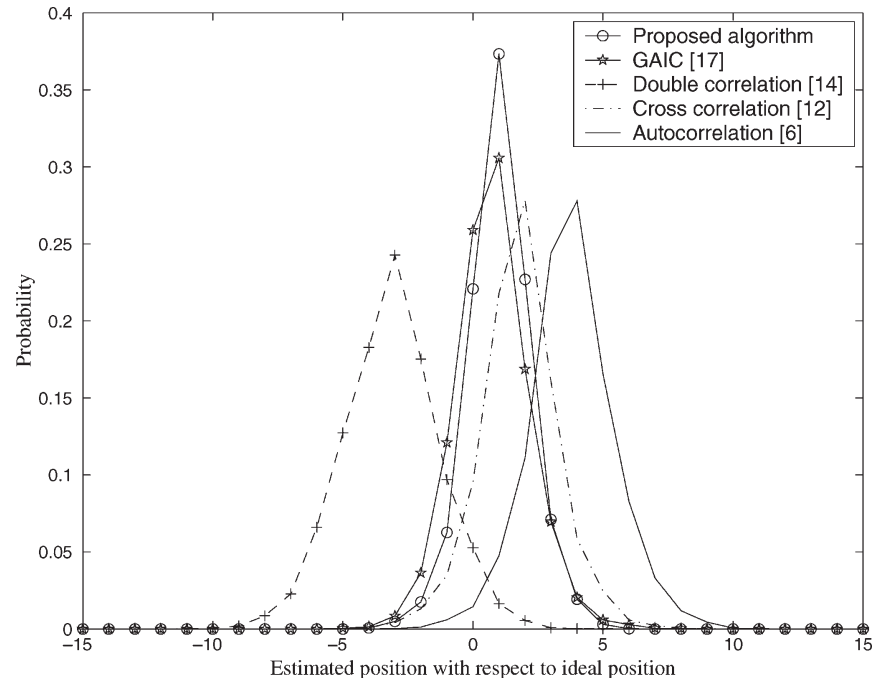

Fig. 6. Distributions of the detected FFT-window starting position for the proposed algorithm and algorithms in [6], [12], [14], and [17] with respect to the ideal FFT-window starting position in channel II.

For other correlation-based algorithms under consideration, although some perform pretty well at certain SNR regions (e.g., autocorrelation algorithm at high SNRs and doublecorrelation algorithm at $5 \mathrm{~dB} \leq \mathrm{SNR} \leq 10 \mathrm{~dB}$ ), in general, they are not as reliable as the proposed algorithm and the algorithm based on GAIC.

Now, let us consider the performances of different algorithms in channel II. Fig. 6 plots the distributions of the estimated FFTwindow positions at $\mathrm{SNR}=30 \mathrm{~dB}$ with respect to the ideal position. Note that the ideal position is the starting point for the FFT window such that (31) is minimized and is different for different channel realizations. First, it can be seen that the distributions for all algorithms have larger variances compared to the case of channel I. This is because the start of the channel is not clear in channel II, so a small amount of noise can lead to a shift in the estimated position. Secondly, from Fig. 6, it is obvious that the proposed algorithm and the algorithm based on GAIC have estimated positions closer to the ideal position compared with the estimates from other algorithms.

Fig. 7 shows the probability of synchronization failure $P_{\mathrm{f}}(0.5 \mathrm{~dB})$ as a function of SNR for channel II. Notice that the curves of $P_{\mathrm{f}}$ in general show an "U shape." This is because at low SNRs, the estimation is not accurate due to the high level of noise, while at high SNRs, although the estimated positions can be quite accurate, a small amount of shift with respect to the ideal position leads to a large amount of loss in SINR [see (28)]. From Fig. 7, it is clear that the proposed algorithm and the algorithm based on GAIC have similar performances and are the best compared to others (at least for SNR $\geq 10 \mathrm{~dB}$ ).

The poor performances of the correlation-based algorithms are mainly due to the fact that the signal filtered through a multipath channel would present a complicated correlation output [as opposed to a single correlation peak in an additive white Gaussian noise (AWGN) channel], making the detection of the correlation peak more difficult. For the proposed algorithm and the algorithm based on GAIC, although the same pilot signal is used, they also incorporate the multipath structure of 


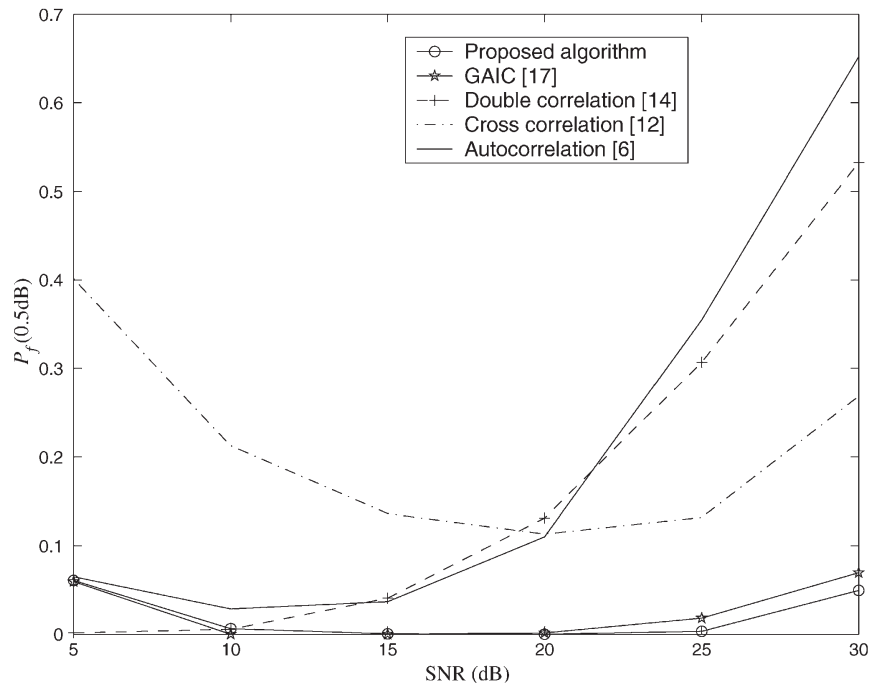

Fig. 7. $P_{\mathrm{f}}(0.5 \mathrm{~dB})$ for the proposed algorithm and algorithms in [6], [12], [14], and [17] as a function of SNR in channel II.

the channel into the system model, making it more robust to multipath fading. Combining the results of Figs. 5 and 7, we can conclude that at medium to high SNRs (SNR $\geq 10 \mathrm{~dB}$ ), joint estimation of the channel and timing (the proposed algorithm and the algorithm based on GAIC) gives better performance than the correlation-based algorithms, although their implementation complexity is much simpler.

Finally, we want to mention that although the performances of the proposed algorithm is comparable to that of the GAIC algorithm, the complexity of the proposed algorithm is much smaller. This can be explained as follows. Both algorithms involve a least-squares fitting in the form of $\|\mathbf{r}-\boldsymbol{\Phi} \mathbf{r}\|^{2}$, where $\mathbf{r}$ is an observation vector in the time domain for the proposed algorithm and is an observation vector transformed into the frequency domain using FFT for the algorithm based on GAIC, and $\boldsymbol{\Phi}$ is some square matrix depending on the parameters to be estimated [compare (38) of this paper with that in [17, eq. (4)]. Since the observation length is only 16 for the proposed algorithm while it is 64 for the GAIC algorithm, the number of multiplications for the proposed algorithm in each hypothesis test is 16 times less than that of the GAIC algorithm. Taking into account the fact that, for the proposed algorithm, the number of hypothesis tests is smaller than that of the GAIC algorithm, and there is no need to transform the observation vector into the frequency domain before least-squares fitting, the proposed algorithm is at least 16 times less complex than the GAIC algorithm.

\section{CONCLUSION}

In this paper, based on the ML principle and the preamble structure of IEEE 802.11a standard, a new symbolsynchronization algorithm for IEEE 802.11a WLANs over frequency-selective fading channels is proposed. A realistic channel model was employed, which includes the effects of the physical channel, filtering, and unknown sampling phase offset. Loss in system performance due to synchronization error was used as a performance criterion. Computer simulations showed that the proposed algorithm exhibits better performance than the correlation-based algorithms. When compared to the algorithm based on GAIC, the proposed algorithm has comparable performance, but with significantly reduced complexity.

\section{APPENDIX}

PROOF OF (38)

The first term of (34) can be easily obtained by plugging (35) and (36) into (33) followed by taking logarithm, and it gives

$$
\begin{aligned}
\ln p\left(\mathbf{r}_{n_{1}} ; \hat{\boldsymbol{\theta}}, i, L\right)= & N \ln N-N \ln \pi-N \ln \\
& \times\left\|\mathbf{r}_{n_{1}}-\mathbf{B}_{i}\left(\mathbf{B}_{i}^{\mathrm{H}} \mathbf{B}_{i}\right)^{-1} \mathbf{B}_{i}^{\mathrm{H}} \mathbf{r}_{n_{1}}\right\|^{2}-N .
\end{aligned}
$$

Consider the second term of (34). Performing the differentiations in (37), we have

$$
\mathbf{I}(\boldsymbol{\theta} \mid i, L)=\frac{1}{\sigma^{2}}\left[\begin{array}{ccc}
2 \Re\left(\mathbf{B}_{i}^{\mathrm{H}} \mathbf{B}_{i}\right) & -2 \Im\left(\mathbf{B}_{i}^{\mathrm{H}} \mathbf{B}_{i}\right) & 0 \\
2 \Im\left(\mathbf{B}_{i}^{\mathrm{H}} \mathbf{B}_{i}\right) & 2 \Re\left(\mathbf{B}_{i}^{\mathrm{H}} \mathbf{B}_{i}\right) & 0 \\
0 & 0 & \frac{1}{\sigma^{2}}
\end{array}\right]
$$

It follows that

$\operatorname{det}(\mathbf{I}(\boldsymbol{\theta} \mid i, L))=\frac{2^{2 L}}{\sigma^{2(2 L+2)}} \operatorname{det}\left(\left[\begin{array}{cc}\Re\left(\mathbf{B}_{i}^{\mathrm{H}} \mathbf{B}_{i}\right) & -\Im\left(\mathbf{B}_{i}^{\mathrm{H}} \mathbf{B}_{i}\right) \\ \Im\left(\mathbf{B}_{i}^{\mathrm{H}} \mathbf{B}_{i}\right) & \Re\left(\mathbf{B}_{i}^{\mathrm{H}} \mathbf{B}_{i}\right)\end{array}\right]\right)$.

Using the result

$$
\operatorname{det}\left(\left[\begin{array}{ll}
\mathrm{A}_{11} & \mathrm{~A}_{12} \\
\mathrm{~A}_{21} & \mathrm{~A}_{22}
\end{array}\right]\right)=\operatorname{det}\left(\mathrm{A}_{11}\right) \operatorname{det}\left(\mathrm{A}_{22}-\mathrm{A}_{21} \mathrm{~A}_{11}^{-1} \mathrm{~A}_{12}\right)
$$

we note that for any square matrix $\boldsymbol{\Sigma}$

$$
\begin{aligned}
& \operatorname{det}\left(\left[\begin{array}{cc}
\Re(\boldsymbol{\Sigma}) & -\Im(\boldsymbol{\Sigma}) \\
\Im(\boldsymbol{\Sigma}) & \Re(\boldsymbol{\Sigma})
\end{array}\right]\right) \\
& \quad=\operatorname{det}(\Re(\boldsymbol{\Sigma})) \operatorname{det}\left(\Re(\boldsymbol{\Sigma})+\Im(\boldsymbol{\Sigma}) \Re(\boldsymbol{\Sigma})^{-1} \Im(\boldsymbol{\Sigma})\right) .
\end{aligned}
$$

Since

$$
\begin{aligned}
& \Re(\boldsymbol{\Sigma})+\Im(\boldsymbol{\Sigma}) \Re(\boldsymbol{\Sigma})^{-1} \Im(\boldsymbol{\Sigma}) \\
& \quad=(\Re(\boldsymbol{\Sigma})-j \Im(\boldsymbol{\Sigma})) \Re(\boldsymbol{\Sigma})^{-1}(\Re(\boldsymbol{\Sigma})+j \Im(\boldsymbol{\Sigma})) \\
& \quad=\boldsymbol{\Sigma}^{*} \Re(\boldsymbol{\Sigma})^{-1} \boldsymbol{\Sigma}
\end{aligned}
$$

it follows that

$$
\begin{gathered}
\operatorname{det}\left(\left[\begin{array}{cc}
\Re(\boldsymbol{\Sigma}) & -\Im(\boldsymbol{\Sigma}) \\
\Im(\boldsymbol{\Sigma}) & \Re(\boldsymbol{\Sigma})
\end{array}\right]\right) \\
=\frac{\operatorname{det}(\Re(\boldsymbol{\Sigma})) \operatorname{det}\left(\boldsymbol{\Sigma}^{*}\right) \operatorname{det}(\boldsymbol{\Sigma})}{\operatorname{det}(\Re(\boldsymbol{\Sigma}))}=(\operatorname{det}(\boldsymbol{\Sigma}))^{2} .
\end{gathered}
$$

Plugging (58) into (53), and then taking the logarithm, we have

$$
\begin{aligned}
& \ln \operatorname{det}(\mathbf{I}(\hat{\boldsymbol{\theta}} \mid i, L)) \\
& \quad=2 L \ln 2-2(L+1) \ln \hat{\sigma}^{2}+2 \ln \left(\operatorname{det}\left(\mathbf{B}_{i}^{\mathrm{H}} \mathbf{B}_{i}\right)\right) .
\end{aligned}
$$


Combining the results of (51) and (59), and dropping the terms irrelevant to optimization in (34), the generalized ML rule follows

$$
\begin{aligned}
\Psi_{1}\left(\mathbf{r}_{n_{1}} ; i, L\right)= & (-N+L+1) \\
& \times \ln \left\|\mathbf{r}_{n_{1}}-\mathbf{B}_{i}\left(\mathbf{B}_{i}^{\mathrm{H}} \mathbf{B}_{i}\right)^{-1} \mathbf{B}_{i}^{\mathrm{H}} \mathbf{r}_{n_{1}}\right\|^{2} \\
& -L \ln 2-\ln \left(\operatorname{det}\left(\mathbf{B}_{i}^{\mathrm{H}} \mathbf{B}_{i}\right)\right) .
\end{aligned}
$$

Note that the columns of $\mathbf{B}_{i}$ are constructed by cyclically shifting the sequence $\mathbf{b}_{0} \triangleq\left[\begin{array}{llll}b_{0} & b_{1} & \cdots & b_{15}\end{array}\right]^{\mathrm{T}}$ with different numbers of shifts. Denote $T_{\mathrm{lc}}^{m}\left(\mathbf{b}_{0}\right)$ as the cyclic left shift of $\mathbf{b}_{0}$ by $m$ places (e.g., $\left.T_{\mathrm{lc}}\left(\mathbf{b}_{0}\right)=\left[\begin{array}{llll}b_{1} & \cdots & b_{15} & b_{0}\end{array}\right]^{\mathrm{T}}\right)$. It follows that the $(l, k)$ th element of $\mathbf{B}_{i}^{\mathrm{H}} \mathbf{B}_{i}$ is given by $(l, k=$ $\left.0,1, \ldots, L_{\mathrm{e}}-1\right)$

$$
\begin{aligned}
{\left[\mathbf{B}_{i}^{\mathrm{H}} \mathbf{B}_{i}\right]_{l, k} } & =\left[T_{\mathrm{lc}}^{i+l}\left(\mathbf{b}_{0}\right)\right]^{\mathrm{H}}\left[T_{\mathrm{lc}}^{i+k}\left(\mathbf{b}_{0}\right)\right] \\
& =\mathbf{b}_{0}^{\mathrm{H}}\left[T_{\mathrm{lc}}^{k-l}\left(\mathbf{b}_{0}\right)\right] \\
& \triangleq R(k-l)
\end{aligned}
$$

where $R(\tau)$ is the periodic autocorrelation function of the sequence $\mathbf{b}_{0}$ with relative offset $\tau$ and is independent of $i$. Therefore, the matrix $\mathbf{B}_{i}^{\mathrm{H}} \mathbf{B}_{i}$ depends only on $L$ and we can set $i=0$ in $\mathbf{B}_{i}^{\mathrm{H}} \mathbf{B}_{i}$. With this result, the generalized ML rule simplifies to

$$
\begin{aligned}
& \Psi_{1}\left(\mathbf{r}_{n_{1}} ; i, L\right)=(-N+L+1) \\
& \times \ln \left\|\mathbf{r}_{n_{1}}-\mathbf{B}_{i}\left(\mathbf{B}_{0}^{\mathrm{H}} \mathbf{B}_{0}\right)^{-1} \mathbf{B}_{i}^{\mathrm{H}} \mathbf{r}_{n_{1}}\right\|^{2}-\xi(L)
\end{aligned}
$$

where $\xi(L) \triangleq L \ln 2+\ln \left(\operatorname{det}\left(\mathbf{B}_{0}^{\mathrm{H}} \mathbf{B}_{0}\right)\right)$.

\section{REFERENCES}

[1] IEEE 802.11, Supplement to IEEE Standard for Information Technology Telecommunications and Information Exchange Between Systems-Local and Metropolitan Area Networks-Specific Requirements-Part 11: Wireless LAN Medium Access Control (MAC) and Physical Layer (PHY) Specifications: High-Speed Physical Layer in the $5 \mathrm{GHz}$ Band, IEEE Std. 802.11a-1999, 1999.

[2] R. van Nee and R. Prasad, OFDM for Wireless Multimedia Communications. Boston, MA: Artech House, 2000.

[3] J. Heiskala and J. Terry, OFDM Wireless LANs: A Theoretical and Practical Guide. Indianapolis, IN: Sams, 2002.

[4] M. Speth, F. Classen, and H. Meyr, "Frame synchronization of OFDM systems in frequency selective fading channels," in Proc. IEEE Vehicular Technology Conf. (VTC), Phoenix, AZ, May 1997, pp. 1807-1811.

[5] M. Speth, D. Daecke, and H. Meyr, "Minimum overhead burst synchronization for OFDM based broadband transmission," in Proc. Global Telecommunications (GLOBECOM), Sydney, Australia, 1998, pp. 3227-3232.

[6] J.-J. van de Beek, M. Sandell, and P. O. Borjesson, "ML estimation of time and frequency offset in OFDM systems," IEEE Trans. Signal Process., vol. 45, no. 7, pp. 1800-1805, Jul. 1997.

[7] D. Lee and K. Cheun, "Coarse symbol synchronization algorithms for OFDM systems in multipath channels," IEEE Commun. Lett., vol. 6, no. 10 , pp. $446-448$, Oct. 2002.

[8] T. M. Schmidl and D. C. Cox, "Robust frequency and timing synchronization for OFDM," IEEE Trans. Commun., vol. 45, no. 12, pp. 1613-1621, Dec. 1997.

[9] S. H. Muller-Weinfurtner, "On the optimality of metrics for coarse frame synchronization in OFDM: A comparison," in Proc. Personal Indoor and Mobile Radio Communications (PIMRC), Boston, MA, 1998, pp. 533-537.
[10] M. Mizoguchi, T. Onizawa, T. Kumagai, H. Takanashi, and M. Morikura, "A fast burst synchronization scheme for OFDM," in Proc. IEEE Int Conf. Universal Personal Communications (ICUPC), Florence, Italy, 1998, pp. 125-129.

[11] T. Keller, L. Piazzo, P. Mandarini, and L. Hanzo, "Orthogonal frequency division multiplex synchronization techniques for frequency-selective fading channels," IEEE J. Sel. Areas Commun., vol. 19, no. 6, pp. 999 1008, Jun. 2001.

[12] A. J. Coulson, "Maximum likelihood synchronization for OFDM using a pilot symbol: Algorithms," IEEE J. Sel. Areas Commun., vol. 19, no. 12, pp. 2486-2494, Dec. 2001

[13] — , "Maximum likelihood synchronization for OFDM using a pilot symbol: Analysis," IEEE J. Sel. Areas Commun., vol. 19, no. 12, pp. 2495-2503, Dec. 2001.

[14] F. Tufvesson, O. Edfors, and M. Faulkner, "Time and frequency synchronization for OFDM using PN-sequence preambles," in Proc. Vehicular Technology Conf. (VTC), Amsterdam, The Netherlands, 1999, vol. 4, pp. 2203-2207.

[15] B. Yang, K. B. Letaief, R. S. Cheng, and Z. Cao, "Timing recovery for OFDM transmission," IEEE J. Sel. Areas Commun., vol. 18, no. 11, pp. 2278-2291, Nov. 2000

[16] K.-W. Yip, T.-S. Ng, and Y.-C. Wu, "Impacts of multipath fading on the timing synchronization of IEEE 802.11a wireless LANs," in Proc. Int. Conf. Communications (ICC), New York, Apr. 28-May 2, 2002, pp. 517-521.

[17] E. G. Larsson, G. Liu, J. Li, and G. B. Giannakis, "Joint symbol timing and channel estimation for OFDM based WLANs," IEEE Commun. Lett., vol. 5, no. 8, pp. 325-327, Aug. 2001.

[18] S. A. Fechtel and H. Meyr, "Improved frame synchronization for spontaneous packet transmission over frequency-selective radio channels," in Proc. Personal Indoor and Mobile Radio Communications (PIMRC), The Hague, The Netherlands, 1994, pp. 353-357.

[19] U. Lambrette, J. Horstmannshoff, and H. Meyr, "Techniques for frame synchronization on unknown frequency selective channels," in Proc. Vehicular Technology Conf. (VTC), Phoenix, AZ, 1997, pp. 1059-1063.

[20] Y. Koo and Y. H. Lee, "A joint maximum likelihood approach to frame synchronization in presence of frequency offset," in Proc. Int. Conf. Communications (ICC), New York, 2002, pp. 1546-1550.

[21] J. G. Proakis, Digital Communications, 3rd ed. New York: McGrawHill, 1995.

[22] S. M. Kay, Fundamentals of Statistical Signal Processing, Vol. 1: Estimation Theory. New York: Prentice-Hall, 1993.

[23] — Fundamentals of Statistical Signal Processing, Vol. 2: Detection Theory. New York: Prentice-Hall, 1998.

[24] H. Meyr, M. Oerder, and A. Polydoros, "On sampling rate, analog prefiltering, and sufficient statistics for digital receivers," IEEE Trans. Commun., vol. 42, no. 12, pp. 3208-3213, Dec. 1994.

[25] P. H. Moose, "A technique for orthogonal frequency division multiplexing frequency offset correction," IEEE Trans. Commun., vol. 42, no. 10 , pp. 2908-2914, Oct. 1994.

[26] J. A. Gansman, M. P. Fitz, and J. V. Krogmeier, "Optimum and suboptimum frame synchronization for pilot-symbol-assisted modulation," IEEE Trans. Commun., vol. 45, no. 10, pp. 1327-1337, Oct. 1997.

[27] M. Speth, S. A. Fechtel, G. Fock, and H. Meyr, "Optimum receiver design for wireless broad-band systems using OFDM-Part I," IEEE Trans. Commun., vol. 47, no. 11, pp. 1668-1677, Nov. 1999.

[28] H. Steendam and M. Moeneclaey, "Analysis and optimization of the performance of OFDM on frequency-selective time-selective fading channel," IEEE Trans. Commun., vol. 47, no. 12, pp. 1811-1819, Dec. 1999.

[29] H. Minn, V. K. Bhargava, and K. B. Letaief, "A robust timing and frequency synchronization for OFDM systems," IEEE Trans. Wireless Commun., vol. 2, no. 4, pp. 822-839, Jul. 2003.

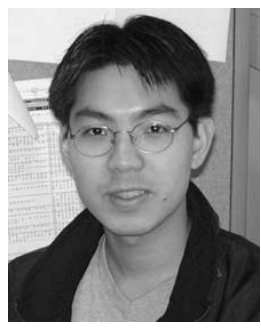

Yik-Chung Wu received the B.Eng. (with honors) and M.Phil. degrees in electronic engineering from the University of Hong Kong, Hong Kong, in 1998 and 2001, respectively, and is currently pursuing the Ph.D. degree at the Texas A\&M University (TAMU), College Station.

From 2001 to 2002, he was a Research Assistant at the University of Hong Kong. His research interests include digital signal processing with applications to communication systems, software radio, and spacetime processing.

Mr. Wu obtained the Croucher Foundation scholarship in 2002 


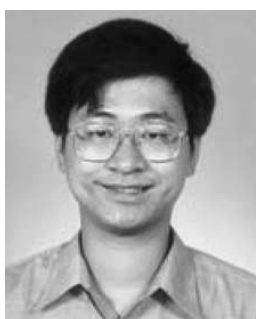

Kun-Wah Yip (M'96) received the B.Eng. (Hons.) and $\mathrm{Ph} . \mathrm{D}$. degrees in electrical engineering from the University of Bradford, Bradford,U.K., in 1991 and the University of Hong Kong, Hong Kong, in 1995, respectively.

From 1995 to 2002, he was with The University of Hong Kong, as a Postdoctoral Fellow and then as a Research Assistant Professor. Currently, he is a member of the Technical Staff at Philips Research East Asia, Shanghai, China. His research interest is on digital TV, wireless local area networks (WLANs), wireless personal area networks (WPANs), orthogonal frequency division multiplexing (OFDM), spread-spectrum techniques, and efficient simulation techniques for communication systems.

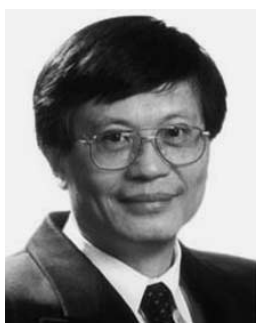

Tung-Sang Ng (S'74-M'78-SM'90-F'03) received the B.Sc.(Eng.) degree from the University of Hong Kong, Hong Kong, in 1972, and the M.Eng.Sc. and Ph.D. degrees from the University of Newcastle, Australia, in 1974 and 1977, respectively, all in electrical engineering.

After graduation, he worked for BHP Steel International and The University of Wollongong, Wollonggong, Australia, for 14 years before he returned to The University of Hong Kong, in 1991, taking up the position of Professor and Chair of Electronic Engineering. He was Head of the Department of Electrical and Electronic Engineering from 2000 to 2003 and is currently Dean of Engineering. His current research interests include wireless communication systems, spread spectrum techniques, code division multiple access (CDMA), and digital signal processing. He has published over 250 international journal and conference papers.

Dr. Ng was the General Chair of International Symposium on Circuits and Systems (ISCAS)' 97 and the VP-Region 10 of IEEE CAS Society in 1999 and 2000. He was an Executive Committee Member and a Board Member of the IEE Informatics Divisional Board (1999-2001) and was an Ordinary Member of IEE Council (1999-2001). He was awarded the Honorary Doctor of Engineering Degree by the University of Newcastle, Australia, in 1997, the Senior Croucher Foundation Fellowship in 1999, the IEEE Third Millenium Medal in 2000, and the Outstanding Researcher Award by The University of Hong Kong in 2003. He is a Fellow of IEE and HKIE.

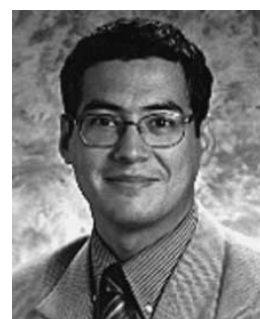

Erchin Serpedin (S'96-M'99-SM'04) received the Diploma of electrical engineering (with highest distinction) from the Polytechnic Institute of Bucharest, Bucharest, Romania, in 1991, the Specialization degree in signal processing and transmission of information from Ecole Superiéure D'Electricité, Paris, France, in 1992, the M.Sc. degree from the Georgia Institute of Technology, Atlanta, in 1992, and the Ph.D. degree in electrical engineering from the University of Virginia, Charlottesville, in January 1999.

From 1993 to 1995, he was an Instructor in the Polytechnic Institute of Bucharest, and between January and June 1999, he was a Lecturer at the University of Virginia. In July 1999, he joined Texas A\&M University (TAMU), Wireless Communications Laboratory, as an Assistant Professor. In 2005, he was promoted to Associate Professor with tenure. His research interests lie in the areas of statistical signal processing and wireless communications.

Dr. Serpedin has received the National Science Foundation (NSF) CAREER Award in 2001, and the Outstanding Faculty Award from TAMU in 2004. He is currently serving as an Associate Editor for the IEEE TRANSACTIONS ON WIRELESS COMMUNICATIONS, the IEEE TRANSACTIONS ON SignAL PROCESSING, the IEEE COMMUNICATIONS LETTERS, the IEEE Signal PRocessing LetTERS, and the IEEE TRANSACTIONS ON COMMUNICATIONS. 